\title{
Model-based design of optical diagnostic instrumentation
}

\author{
Chih-Wen Kan ${ }^{1}$, Kort Travis ${ }^{2}$, James Salazar ${ }^{1}$, Konstantin Sokolov ${ }^{3}$, and Mia K. Markey ${ }^{1,3}$ \\ ${ }^{1}$ Department of Biomedical Engineering, The University of Texas at Austin, Austin, Texas, \\ U.S.A.; \\ ${ }^{2}$ Department of Physics, The University of Texas at Austin, Austin, Texas, U.S.A.; \\ ${ }^{3}$ Department of Imaging Physics, The University of Texas M.D. Anderson Cancer Center, \\ Houston, Texas, U.S.A.
}

\begin{abstract}
Biophotonics methods are attractive since they allow for the non-invasive diagnosis of cancer. Experiments were carried out to investigate the feasibility of detecting early pre-cancer using optical spectroscopy. However, optimization of instrumentation design parameters remains challenging because of the lack of metrics to evaluate the performance of certain design parameters. For example, although using angled-collection geometry has been shown to collect depth sensitive spatial origins, the performance of devices with angled-collection geometries are not well characterized or quantified. In this study, we use a polarization-sensitive Monte Carlo simulation (Pol-MC) to aid in the design of instrumentation for the early detection of epithelial cancer. The tissue is modeled in layers: (0) air outside the tissue, (1) epithelial layer, (2) thin pre-cancer layer of cells, (3) thin basement membrane, implemented as a thin transparent layer, and (4) the stroma, implemented as a thick layer of scattering material. We propose a new metric, Target Signal Ratio (TSR), to evaluate the proportion of signal that is scattered from a target layer, which is the basal/pre-cancer layer. This study is a proof-of-concept for the application of computational techniques to facilitate instrument design.
\end{abstract}

Keywords: model-based, Monte Carlo, simulation, layer thickness, target signal ratio, TSR

\section{INTRODUCTION}

Probing biological tissue, a scattering medium, with light, has many potential applications in biomedical diagnostics. Cancerous cells and normal cells have very different morphologies, largely because cancerous cells have fast, generally uncontrolled growth processes. These morphological differences also affect the way these cells interact with light; for example, it was found that the difference (e.g., in scattering/absorption coefficients) between the optical properties of normal and cancerous tissues results in a detectable difference in their reflectance spectra. For these reasons, biophotonics methods provide the promise of early diagnosis of cancer in a relatively inexpensive and non-invasive fashion.

Polarized light sources, and polarization-sensitive detectors, have been used to provide better separation between shallow and deep signals collected from tissue. This is highly advantageous as cancer generally originates near the surface layers of organs, and therefore, statistics with more diagnostic relevance will be obtained from these enhanced superficial signals. For example, polarized incident light and polarization-enabled collection fibers have been widely used to probe the structure of superficial tissue. This superficial signal enhancement is due largely to the fact that multiply scattered light preserves the incident polarization, while singly scattered light does not. It has been shown both experimentally and in simulation that polarized light is useful in studying tissue, and that tissue properties can be extracted through changes in the light's polarization state. ${ }^{1-9}$

When light is used for diagnosis, it is important to ensure that it travels through target areas where cancer and pre-cancer are located. Several fundamental questions must be addressed in order to evaluate the effectiveness of the use of polarization for diagnostic purposes. For example: What are the specific signals collected by parallel and perpendicular polarizers? Is the penetration depth of the light significantly different for multiple layer

Further author information:

C. W. Kan: Email: wendykan@gmail.com, M. K. Markey: Email:mia.markey@mail.utexas.edu

Biomedical Applications of Light Scattering V, edited by Adam P. Wax, Vadim Backman,

Proc. of SPIE Vol. 7907, 79070I · C 2011 SPIE · CCC code: 1605-7422/11/\$18 - doi: 10.1117/12.875885

Proc. of SPIE Vol. 7907 79070I-1 
models? What is the relationship between penetration depth and pathlength? Can signals be discriminated through the use of singly scattered and multiply scattered light? And most importantly, is the instrument actually effectively collecting the signal from the target layer, which is the precancer layer? Answers to these questions are critical for the development of new diagnostic instrumentation, as well as the validation of existing instrumentation designs. Particularly important are considerations of how probe geometry affects the collection of light.

In this study, we use a polarization-sensitive Monte Carlo simulation (Pol-MC) to aid in the design of instrumentation for the early detection of epithelial cancer. In contrast to other Monte Carlo implementations, which primarily use the Henyey-Greenstein phase function, the Pol-MC numerical code accurately calculates scattering phase functions based on comprehensive Mie theory calculations, and fully retains polarization information at both scattering and interface transitions. Through the analysis of simulation results, we compared the use of angled and flat detector geometries in a hypothetical diagnostic instrument. Further, by taking advantage of the stochastic nature of the Monte Carlo technique itself, we are able to evaluate information not normally accessible through any physical measurement; for example, we are able to evaluate the Target Signal Ratio (TSR) achievable by the instrument through the sub-classification of scattered photon paths, as represented in the simulation, and this information is also applicable to assist in the development of algorithms for the analysis of the instrument's measurements. These promising results provide a proof-of-concept for the application of this type of stochastic computational technique to diagnostic instrument design.

\section{METHODS}

\subsection{Polarization sensitive Monte Carlo simulations (Pol-MC)}

The numerical simulation of polarization sensitive Monte Carlo is performed with a code based on that originally developed by Dr. Daniel Côté, ${ }^{10}$ with several implementation extensions developed by the authors of this study.

Côté's ${ }^{10}$ original implementation of Pol-MC did not allow the convenient simulation of complex multilayer structures. Figure 1 shows a graphic illustration of how this "touching" of layers is accomplished. Accomplished in our study, both layer objects have two surface elements: one on top and one on the bottom. When the two surface elements are detected to be at the same location, their ObjectOuside are modified so that they correctly point to the adjacent layer.

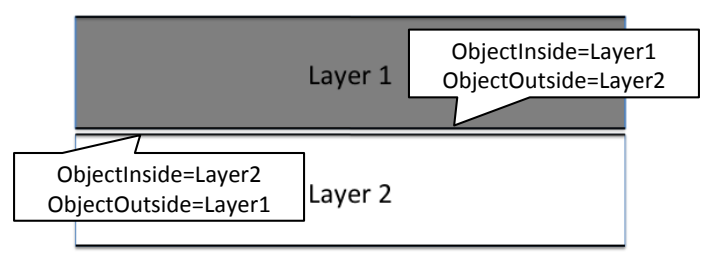

Figure 1. Illustration of how we handle layers "touching" each other.

\subsection{Target Signal Ratio (TSR) analysis}

A unique advantage of using Monte Carlo simulation is that it produces a complete list of photon traveling paths, i.e., the position, weight, and polarization state of every photon at every step at every move through the simulation geometry. This list of photon traveling record is not easily accessible experimentally, if at all.

In this study, we define a measure, Target Signal Ratio (TSR), as an indicator of whether a given design is feasible. Here we define the target signal as photons detected from a specific layer of tissue via scattering processes.

The Target Signal Ratio (TSR) is calculated as

$$
T S R_{k}=\frac{\Phi_{k}}{I-\Phi_{k}}
$$

where $I$ is the total intensity, $k$ is the "target" index of the photon category, and $\Phi_{k}$ is the surface fluence at the detector surface for all photons from category $k$. Fluence is the integral of the radiance over all directions. ${ }^{11}$ 


\subsection{Simulation parameters}

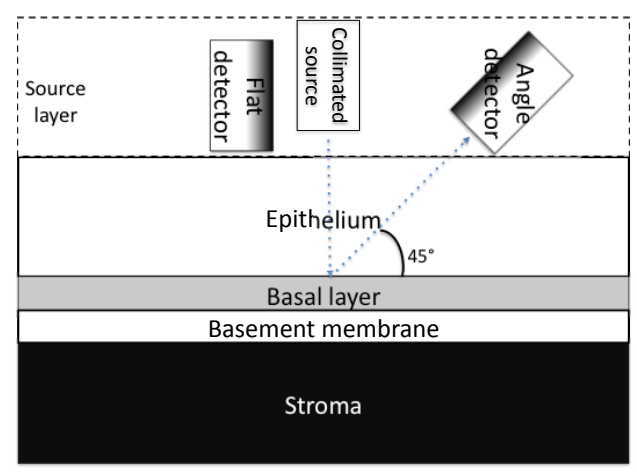

Figure 2. Illustration of the geometry setup in our simulation. Photons enter the tissue from a collimated source. The tissue consists of four layers: epithelium, basal layer, stromal membrane, and stroma. These four layers are infinite layers and they touch each other with no gaps in between. Two detectors are placed above the epithelium with very small gaps. The angle detector has a $45^{\circ}$ angle with the epithelium, while the flat detector is aligned with the surface of the epithelium. Note that a source layer is placed on top of the epithelium layer both to provide some degree of index-matching and for computational purposes.

In our simulation, the geometry is set up as illustrated in Fig. 2. A four-layer model is simulated: (1) epithelial layer, (2) thin basal layer of cells, (3) basement membrane, implemented as a thin transparent layer, (4) stroma, implemented as a thick layer of scattering material. We also have a collimated light source with photons entering the tissue perpendicular to the surface. Two detectors are placed above the epithelium: The angle detector has a $45^{\circ}$ angle with the epithelium, while the flat detector is aligned with the surface of the epithelium.

In the simulation, the optical properties are defined in the input XML files. The wavelength used is $633 \mathrm{~nm}$, all simulation results shown (excepting the wavelength-series) are simulated with $1.28 \times 10^{9}$ photons. Tab. 1 and Tab. 2 show some of the most important optical properties selected in this study. These parameters are chosen carefully to match the values reported in literature, on the conservative side. ${ }^{11}$ The properties that we have used for the Stromal membrane are selected to be consistent with those of other biological membranes, although these properties are plausible we believe that the specific properties of this membrane may be unknown.

Table 1. Tissue properties used for this study. *Note that some of the parameters vary between different runs, the layer thickness are specified in Tab. 2. Units in lengths are in $\mu m .{ }^{* *} \mathrm{HG}$ : Henyey-Greenstein.

\begin{tabular}{|c|c|c|c|c|c|c|c|c|}
\hline & thickness & scattering & $\mathrm{g}$ & index(media) & index(scatterer) & $\mu_{a}$ & $\mu_{s}$ & scatterer radius \\
\hline Source layer & 10000 & $\mathrm{HG}^{* *}$ & 0.5 & 1.335 & - & 0.1 & 1 & - \\
\hline Epithelium & varies & Jaillon & - & 1.369 & 1.41 & 1 & 100 & 6 \\
\hline Basal layer & varies & Jaillon & - & 1.369 & 1.41 & 1 & 120 & varies \\
\hline $\begin{array}{c}\text { Basement } \\
\text { membrane }\end{array}$ & 30 & $\mathrm{HG}^{* *}$ & 0.5 & 1.41 & - & 1 & 0 & - \\
\hline Stroma & 1000 & Jaillon & - & 1.369 & 1.41 & 1 & 150 & 6 \\
\hline
\end{tabular}

\section{RESULTS}

\subsection{Scattering event distribution}

We generate the total fluence plots to check the scattering distribution spatially. The total scaled fluence for Model 4 is shown in Fig. 3(a). In the figure, we can interpret the intensity as the density of scattering events in 
Table 2. Tissue layer thickness used in this study. Four sets of simulation parameters are presented here.

\begin{tabular}{|c|c|c|c|c|}
\hline thickness and radius $(\mu \mathrm{m})$ & Model 1 & Model 2 & Model 3 & Model 4 \\
\hline \hline Source layer & 10000 & 10000 & 10000 & 10000 \\
\hline Epithelium & 250 & 250 & 230 & 190 \\
\hline Basal layer & 20 & 20 & 40 & 80 \\
\hline Stromal membrane & 30 & 30 & 30 & 30 \\
\hline Stroma & 1000 & 1000 & 1000 & 1000 \\
\hline Basal layer scatterer radius & 6 (normal) & 8 (abnormal) & 8 (abnormal) & 8 (abnormal) \\
\hline
\end{tabular}

each location. The basal layer has a large number of scattering events both because it is closer to the source, and because its scattering coefficient $\left(\mu_{s}\right)$ is $120 \mathrm{~cm}^{-1}$, is slightly higher than that of the epithelium which is $100 \mathrm{~cm}^{-1}$. The dark gap between the basal layer and the stroma corresponds to the stromal membrane; with $\mu_{s}$ of zero, the scaled fluence for this region is also zero. Finally, although we would expect the stroma to have high scaled fluence because it is highly scattering $\left(\mu_{s}=150\right)$, it does not show significantly high intensity in the figure; this is mostly because there are less photons that enter the stroma.

With respect to the scattering intensity in the stroma, this idea is borne out by comparison between the two models; if we compare Model 4 (Fig. 3(a)) with Model 2 (Fig. 3(b)), with all the parameters being the same except the basal layer thickness, we find the scaled fluence in the stroma much higher in Model 2 than in Model 4. This shows that because of the thicker basal layer, and also due to the more complicated scattering geometry, fewer photons are likely to penetrate through the basal layer and enter stroma in Model 4.

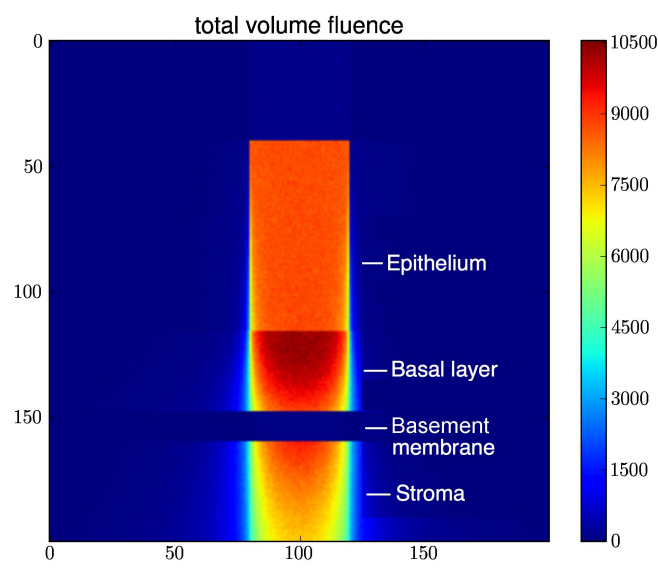

(a)

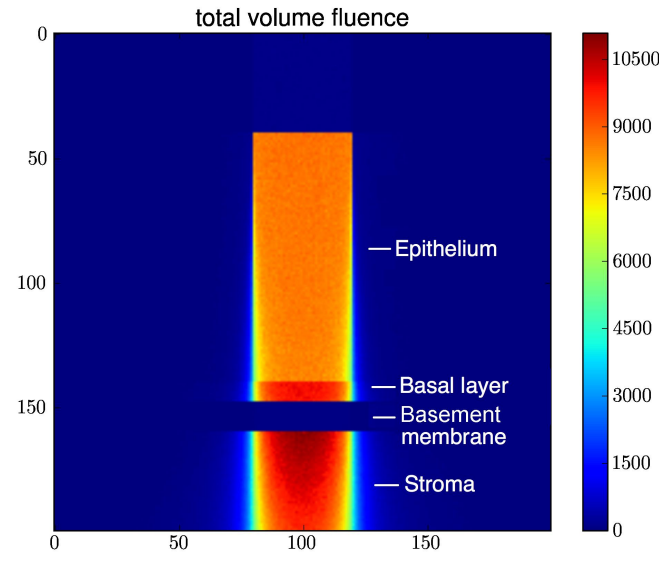

(b)

Figure 3. Total fluence for Model 4 (Fig. 3(a)) and Model 2 (Fig. 3(b)). Photons enter the tissue from the top. These volume fluence plots represent the total spatial scattering density. The basal layers in both models have high fluence and show a clear boundary between epithelium and basal layer. Stromal membranes do not scatter, so they have a dark color in these figures. Stroma layers are also highly scattering, but the intensity also depends on how many photons actually enter the layers. Since Model 4 has a thick basal layer, fewer photons enter the stroma and less scattering is indicated.

\subsection{Studying detector angles}

In order to study instrument design details relating to the specifics of detection, we classified the photon traveling paths and plotted the fluence plots only for photons that are collected by a given detector. This method allows us to specifically evaluate the photons detected, which means it effectively allows us to analyze where scattering events have happened, after the fact of detection, for detected photons. Fig. 4(a) and Fig. 4(b) are two examples of fluence plots from detectors, one from the angle detector and one from the flat detector. We see very clearly from both images that the photons form two beams: one going into the tissue, and one scattered near the bottom of epithelium and in basal layer. We can also tell from these figures that the fluence is always most significant 
where the two beams overlap. This means that since the flat detector is placed right next to the source, its distance from the incident beam is smaller, allowing more overlap between the beams. Since the difference between these two fluence plots difference is largely caused by this difference in distances between the detectors and the light source, it suggests that the rotated angle of the detector is not as important a factor as the distance from the source. We find this conclusion to be particularly interesting, as it is counter-intuitive to analysis based strictly on ray-tracing considerations.

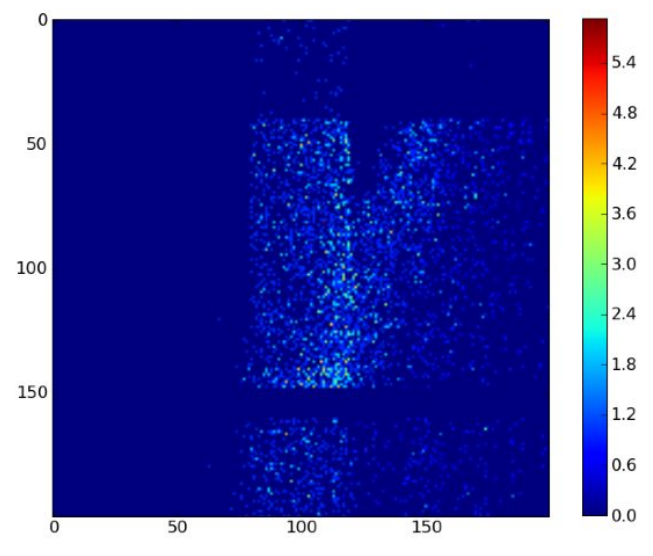

(a)

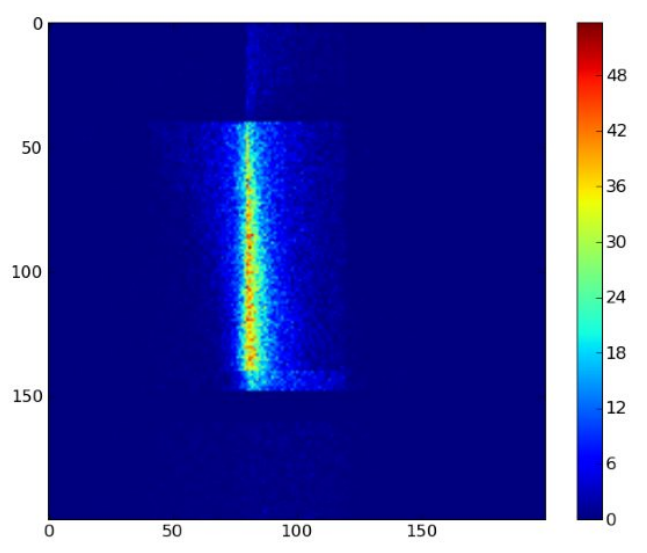

(b)

Figure 4. Fluence for Model 1 with an angle-detector(Fig. 4(a)) and flat-detector (Fig. 4(b)). This image shows the fluence associated with all photons collected by a detector. The photons form two beams: one going into the tissue, and one scattered from the bottom of epithelium and in the basal layer. The fluence is always most significant when the two beams overlap. This plot is for uncollimated detectors, which means all photons arriving at the detector are collected, regardless of their incident angle at the detector surface.

\subsection{Detectors with collimation}

Collimated light is light traveling in a nearly parallel direction. For a source in a Monte Carlo simulation, which can be seen as a type of ray-optics computation, a collimated source is often perfectly collimated, that is, it is comprised of exactly parallel rays, with no divergence at all. This means that any spread in the beam diameter with propagation is associated with either scattering events, or with interface transitions. We now study the effects of collimating the detectors. In simulation, this is done by adjusting the acceptance angle range for photons incident on the detector. In a physical measurement, this selection is automatically done due to the numerical aperture (NA) of the physical detector. In Fig. 5(a) and Fig. 5(b), we show the fluence plots of photons that scatter from the basal layer, detected by an angle detector. In Fig. 5(a), we can clearly see many photons that have scattering events right below the detector but not on the straight line path. These scattered photon are eliminated in Fig. 5(b) with a collimated detector. These results further confirm our speculation in Section 3.2 that if detector collimation is not considered the detectors would perform similarly regardless of rotation angles. However, when the detectors are collimated, which here specifically means when we consider only photons with acceptance angles with minimum acceptance cosine $=0.95$, fewer photons are collected by the detector, and further, photons that carry information not relevant to the target layer are eliminated by the collimated detectors. This minimum acceptance cosine of 0.95 is equivalent to a numerical aperture (NA) of 0.35 , which is within the normal range for optical fibers.

\subsection{TSR analysis}

TSR can be calculated by classifying the photon travel paths in order to determine if the signals collected by detectors were actually from the target region of interest. Here we present TSR from an angled detector, with the signal defined as photons scattering from the basal layer. From Tab. 3 and Tab 4, we can see that TSR 


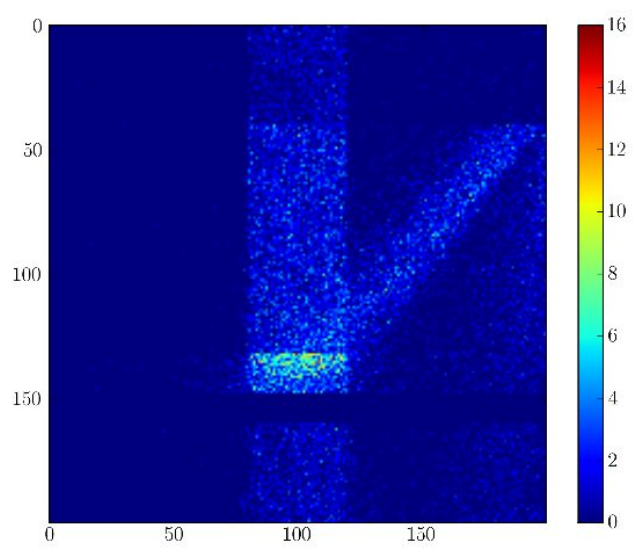

(a)

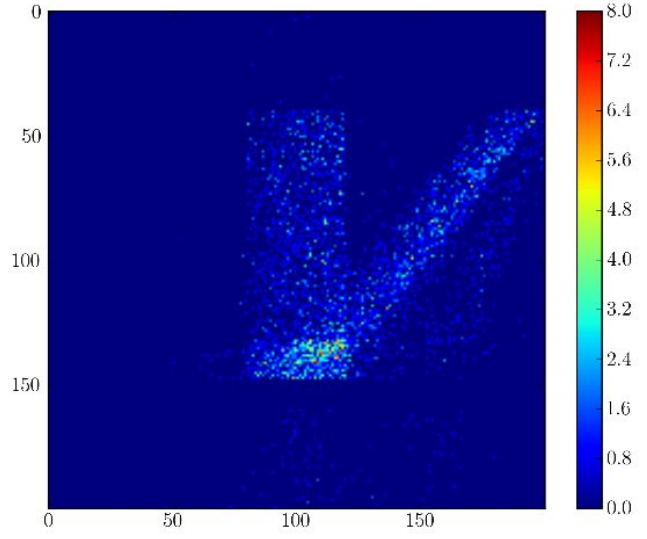

(b)

Figure 5. Fluence for Model 1 with an uncollimated angle-detector(Fig. 4(a)) and collimated angle-detector (Fig. 4(b)). These images show the fluence caused by all the photons detected by a detector. The collimated detector accepts photons that have acceptance cosine values between 0.95 and 1 , whereas the uncollimated detector accepts all photons that hit the detector, regardless of acceptance cosine. These figures show that collimated detectors eliminate many photons that scatter from the stroma and other undesirable locations, and that they enhance the collection of photons scattered from the basal layer.

Table 3. TSR calculation for an angle detector: collimated (NA = 0.35). All four models are used here. Columns labeled as Epithelium, Basal, and Stroma are the numbers of photons that are scattered from these layers. The "target" used in TSR calculation is the basal layer.

\begin{tabular}{|c|ccc|c|}
\hline & Epithelium & Basal & Stroma & Collimated TSR \\
\hline Model 1 & 8986 & 33158 & 1746 & 3.0895 \\
\hline Model 2 & 636 & 7007 & 1192 & 3.8328 \\
\hline Model 3 & 558 & 13459 & 11567 & 7.8490 \\
\hline Model 4 & 385 & 23491 & 1003 & 16.9213 \\
\hline
\end{tabular}

for collimated detectors are significantly higher than TSR for uncollimated detectors as much as 12-25 times higher. A higher value of TSR is resulted from a higher fraction of collected photons scattered from the target layer of interest, which implies the instrumentation collects more diagnostic information of target. This result agrees with the results in Section 3.3 that collimated detectors remove unwanted noise associated with excessive scattering from outside the region of interest.

Also, in Tab. 3 and Tab. 4, we see that the thickness of the target layer (in this case, basal layer) dominates TSR. Model 4 has the thickest basal layer of $80 \mu \mathrm{m}$, while that of Model 1 and 2 is 20 , and Model 3 is 40 . An additional factor for TSR may be related to the scatterer size. The difference between Models 1 and 2 is the scatterer size of their basal layers. Model 2 has an abnormal scatterer size of $8 \mu m$, while Model 1 is 6 $\mu \mathrm{m}$. However, due to the combined interactions of increasing basal layer thickness, and increasing scatterer size, which are present in these simulations, the isolated effect of either factor alone cannot be determined without

Table 4. TSR calculation for an angle detector: uncollimated (NA = 1). All four models are used here. Columns labeled as Epithelium, Basal, and Stroma are the numbers of photons that are scattered from these layers. The "target" used in TSR calculation is the basal layer.

\begin{tabular}{|c|ccc|c|}
\hline & Epithelium & Basal & Stroma & Uncollimated TSR \\
\hline Model 1 & 163053 & 51622 & 43415 & 0.2500 \\
\hline Model 2 & 111217 & 21343 & 25490 & 0.1561 \\
\hline Model 3 & 110727 & 42719 & 23006 & 0.3194 \\
\hline Model 4 & 108527 & 87431 & 19167 & 0.6847 \\
\hline
\end{tabular}


further simulations.

\section{DISCUSSIONS AND CONCLUSIONS}

Tissue optics has demonstrated its potential in recent years, and various experimental instrument designs have been developed to non-invasively measure signals specific to precancer diagnosis. However, whether the instrument is actually specifically probing the desired signal or not has remained an open question. Researchers indirectly measure instrument parameters such as depth sensitivity, but there is no standard metric to use in instrument design. This fact is particularly evident when considering complex photon path behavior in scattering media, where it is not at all evident that considerations based on simple ray-optics estimates, or analogous measurement, are applicable.

Pol-MC is not only a simulation of light propagating in biological tissue, but also a tool that provides additional, highly valuable information relating to the history of the photon travel paths, including polarization. For example, by classifying photon paths into various categories, it is possible to understand where the scattering events have the highest density, and the associated concept of the TSR allows us to evaluate whether a given source / detector configuration is collecting from the region of interest. All of this information has great potential to offer investigators a way to understand the full degree of interaction of light with biological tissue, and in addition it offers a standard tool to evaluate multiple objectives in instrument design. Furthermore, as most of the widely-used Monte Carlo simulations use the unpolarized Henyey-Greenstein phase function as the scattering angle sampling mechanism, Pol-MC offers a much more realistic model for scattering, since it includes higher order Mie scattering, with full polarization information.

The simulation parameters used in this study are for general purposes and not intended for a specific type of experimental design. It should be noted that with respect to effectively using this type of simulation information, it is perhaps even more important than it normally is that the model parameters accurately represent what is being simulated. For example, with respect to simulation of biological tissue specifically, although much information is available, almost as much still remains to be discovered. ${ }^{11}$

Our study of comparing angled- and flat-detectors shows that for uncollimated detectors, the rotation angle of the detector does not really affect the collection efficiency. However, by adjusting the acceptance angle for photon incidence, a collimated detector can be simulated. And in this case, specifically when NA $=0.35$, the angled-detector is able to eliminate a significant fraction of the photons that are scattered from locations outside the region of interest. The results from TSR analysis confirmed this: the collimated detector has TSRs that are much higher than those for the uncollimated detector. This observation is in correspondence to physical intuition, as angled detectors aim at the region overlapping with source illumination; ${ }^{12}$ however, there are fine details of the behavior that are counter-intuitive to expectations based solely on a ray-optics analysis.

This simulation tool can be extended to consider various modalities. Considering its ability to provide information such as polarization states of the photon, it can be used to study effects caused by changes of optical properties. Our analyses provide a platform for comparing optical property changes: fluence plots and TSR can be used as metrics to study changes of optical parameters. This "virtual probe design" tool may guide future clinical applications for detection of epithelial cancer.

\section{ACKNOWLEDGMENTS}

The help from Dr. Daniel Côté is greatly appreciated for our using and modifying of Pol-MC. The authors would also like to acknowledge the Texas Advanced Computing Center (TACC) at The University of Texas at Austin for providing HPC resources that have contributed to the research results reported within this paper. URL: http: //www.tacc.utexas.edu

\section{REFERENCES}

1. K. Sokolov, R. Drezek, K. Gossage, and R. Richards-Kortum, "Reflectance spectroscopy with polarized light: is it sensitive to cellular and nuclear morphology," Optics Express 5(13), pp. 302-317, 1999. 
2. K. Sokolov, L. Nieman, A. Myakov, and A. Gillenwater, "Polarized reflectance spectroscopy for pre-cancer detection.," Technol Cancer Res Treat 3(1), pp. 1-14, 2004.

3. M. Antonelli, A. Pierangelo, T. Novikova, P. Validire, A. Benali, B. Gayet, and A. De Martino, "Mueller matrix imaging of human colon tissue for cancer diagnostics: how monte carlo modeling can help in the interpretation of experimental data," Optics Express 18(10), pp. 10200-10208, 2010.

4. X. Wang, L. V. Wang, C.-W. Sun, and C.-C. Yang, "Polarized light propagation through scattering media: time-resolved monte carlo simulations and experiments," Journal of Biomedical Optics 8(4), pp. 608-617, 2003.

5. S. Jacques, J. Roman, and K. Lee, "Imaging superficial tissues with polarized light," Lasers in Surgery and Medicine 26(2), pp. 119-129, 2000.

6. J. Mourant, A. Hielscher, A. Eick, T. Johnson, and J. Freyer, "Evidence of intrinsic differences in the light scattering properties of tumorigenic and nontumorigenic cells," Cancer 84(6), pp. 366-374, 1998.

7. G. Zonios, L. Perelman, V. Backman, R. Manoharan, M. Fitzmaurice, J. Van Dam, and M. Feld, "Diffuse reflectance spectroscopy of human adenomatous colon polyps in vivo," Appl. Opt 38(31), pp. 6628-6637, 1999.

8. A. Hielscher, J. Mourant, and I. Bigio, "Influence of particle size and concentration on the diffuse backscattering of polarized light from tissue phantoms and biological cell suspensions," Applied optics 36(1), pp. 125$135,1997$.

9. X. Wang, G. Yao, and L. Wang, "Monte carlo model and single-scattering approximation of the propagation of polarized light in turbid media containing glucose," Applied optics 41(4), pp. 792-801, 2002.

10. D. Côté and I. Vitkin, "Robust concentration determination of optically active molecules in turbid media with validated three-dimensional polarization sensitive monte carlo calculations," Optics Express 13(1), pp. 148-163, 2005.

11. A. Welch, M. Gemert, et al., Optical-Thermal Response of Laser-Irradiated Tissue, Springer, 1995.

12. L. Nieman, M. Jakovljevic, and K. Sokolov, "Compact beveled fiber optic probe design for enhanced depth discrimination in epithelial tissues," Opt. Express 17, pp. 2780-2796, 2009. 